\title{
Memory integration in the autobiographical narratives of individuals with autism
}

\author{
Rachel S. Brezis * \\ Sagol Center for Applied Neuroscience, School of Psychology, Interdisciplinary Center, Herzliya, Israel \\ ${ }^{*}$ Correspondence: brezisrs@gmail.com \\ Edited by: \\ Dorit Ben Shalom, Ben Gurion University, Israel \\ Reviewed by: \\ Dermot Bowler, City University, UK \\ Valérie Chaput, Hôpital Robert Debré, France
}

Keywords: autism spectrum disorder, autobiographical memory, episodic memory, integrative functions, self, personal narratives, medial prefrontal cortex, memory specificity

\section{INTRODUCTION}

As part of a unifying theory of autism, Ben Shalom (2009) proposed that while procedural, perceptual and semantic memory functions are intact in Autism Spectrum Disorder (ASD), the more integrative level of episodic memory is impaired. According to Ben Shalom, this reduced integration may be due to the reduced function of the medial prefrontal cortex (mPFC), which may also explain the reduced integration found in motor, sensory-perceptual and emotional processes in ASD. The present review examines this hypothesis, by focusing on evidence regarding autobiographical memory (AM) episodes in ASD — arguably the highest form of memory integration processes.

Most research on memory in ASD thus far has focused on memory for experimentally-presented stimuli (Lind, 2010; Boucher et al., 2012). The present paper builds on this literature to examine the rich evidence that has recently accumulated from in-depth, systematic studies of AM in ASD-memories of personally-related events that are naturalistically accumulated over a person's lifetime. Of note, research on AM is limited in its focus on memories that cannot be as readily verified (but see Bruck et al., 2007), and in its reliance on high-functioning verbal individuals. Nonetheless, studies of AM provide us with an unparalleled perspective on the naturalistic process of

Abbreviations: AM, Autobiographical Memory; ASD, Autism Spectrum Disorders; AS, Asperger Syndrome; mPFC, medial Prefrontal Cortex; TD, Typically Developing. memory integration in ASD. Specifically, this review aims to determine how well memory episodes are integrated in ASD; which elements become integrated and which do not; whether the ability to form integrated, episodic memories relates to other cognitive and emotional capacities; and how this pattern of integration changes over time.

\section{SEMANTIC AND EPISODIC AUTOBIOGRAPHICAL MEMORY (AM)}

The declarative memory system comprises semantic and episodic components. Semantic memories are memories of timeless, de-contextualized facts. Episodic memory refers to personal events recollected in the context of a particular time and place, with some reference to oneself as a participant in the episode (Tulving, 2002). Thus, episodic memories involve two functions: the ability to bind different perceptual elements; and, in humans, the ability to perceive of oneself within this context. On a neurobiological level, episodic memory storage and retrieval are thought to involve the interaction of cortical association areas, in which basic sensory information regarding what occurred and where is stored; the hippocampus, which binds these elements into cohesive memories of individual events; and the $\mathrm{mPFC}$, which further contextualizes these events into schemas, such as the self (Preston and Eichenbaum, 2013).

AM refers to memory for information pertaining to the self; and while it is often viewed as overlapping with episodic memory, the two are not synonymous (Gilboa, 2004). Episodic memory is a memory system, while AM is a type of content (Gardiner, 2008). Thus, episodic memory functions can encompass both AM and simple phenomena that do not necessarily represent self-relevant information (e.g., source memory). At the same time, AM in fact comprises of both semantic and episodic knowledge (e.g., semantic knowledge of one's date of birth, alongside an episodic memory of one's last birthday).

In children with ASD, both semantic and episodic AM is reduced (Bruck et al., 2007; Bon et al., 2012; Goddard et al., 2014), though by adulthood, adults with ASD show a spared memory for semantic AM, alongside reduced episodic AM (Klein et al., 1999; Crane and Goddard, 2008). These studies suggest that as semantic AM may grow in ASD, episodic AM impairments are pervasive. These results fit with the general memory profile in ASD, viz., spared semantic memory alongside difficulties with episodic memory, which is found across experimental studies (Boucher and Bowler, 2008). The present review concerns itself primarily with episodic AM in ASD, though semantic memory will be discussed as it relates to the content of autobiographical narratives.

\section{EPISODIC SPECIFICITY AND NARRATIVE INTEGRATION}

The most common marker of successful episodic AM integration is its degree of specificity: to what extent is the memory vivid? Memory specificity is considered a marker of hippocampus and mPFC re-engagement during memory retrieval (Piolino et al., 2009). Memories are coded 
as "specific" if, in response to a cue, participants provide a narrative that is specific in time and place (e.g., "on my last birthday I went to Yogurtland with friends"); rather than a vague or repetitive occurrence. Using several variants of this task, autobiographical narratives in ASD have consistently been shown to be reduced in specificity, compared with control participants, in every reported study (Goddard et al., 2007, 2014; Crane and Goddard, 2008; Crane et al., 2009, 2010, 2012, 2013; Tanweer et al., 2010; Brezis et al., 2012; Chaput et al., 2013; Maister et al., 2013). In some cases, AM retrieval in individuals with ASD than is also slower and more effortful than for control participants (Goddard et al., 2007; Chaput et al., 2013).

More broadly, the narrative structure of AM in both children and adults with ASD has been found to be reduced in integration. The personal narratives of adults with ASD are less likely to have an organizing high-point and resolution (McCabe et al., 2013); and those of children with ASD are more likely to resemble a list of actions or descriptions than a goal-directed sequence (Goldman, 2008). Furthermore, both children and adults with ASD employ fewer causal connectors and evaluations and less complex syntax in their personal narratives than TD controls (Losh and Capps, 2003, 2006; King et al., 2013; McCabe et al., 2013). Together, these studies point to a general impairment of AM integration in ASD, manifest both in reduced specificity and narrative structure.

\section{MEMORY CONTENT}

The richness of autobiographical narrative data allows an examination not just of what is missing from the personal memories of individuals with autism, but also of what they include. Early studies found that both the published and experimentallyinduced autobiographical narratives of adults with autism included very concrete, visually-oriented reports (Hurlburt et al., 1994; Frith and Happé, 1999); and this finding was further replicated in a study of children's narratives (Losh and Capps, 2006; but see Boucher, 2007 and Ben Shalom et al., 2010 for a conflicting case study). Indeed, these findings fit with Ben Shalom's (2009) claim that perceptual memory, presumably subserved by a network including the rhinal cortex, is unimpaired in autism.

Further studies of the content of personal memories in ASD found striking differences in the topics raised by individuals with ASD and TD controls. For instance, youth with ASD are less likely to mention humans (e.g., family members), and more likely to mention non-humans, than TD youth (Brezis et al., 2012; Chaput et al., 2013). Given that difficulties in socialemotional processing are considered a core symptom of ASD, it is not surprising that individuals with autism have a reduced memory for social and emotional content (Souchay et al., 2013; Brezis et al., 2014). Beyond their reduced mentions of self and others, children and adults with ASD consistently make fewer evaluations regarding their own, or others' mental states (Losh and Capps, 2003, 2006; Brezis et al., 2012; Brown et al., 2012; Bang et al., 2013; King et al., 2013).

The reduced focus on self and others and increased focus on fictional characters in the personal narratives of individuals with autism is echoed in naturalistic, ethnographic studies of autism. During dinnertime conversations, youth with autism are more likely to spontaneously recount a pre-existing narrative they read or viewed, than a personal event they experienced, compared with their TD interlocutors (Solomon, 2004). Furthermore, ASD individuals' personal interests in finance, dinosaurs, and religious narratives, or even their tendency to hoard, can become woven into their identities and sense of self (Nickrenz, 2007; Sirota, 2010; Brezis, 2012; Skirrow et al., 2014). Returning to Ben Shalom's hypothesis, spared semantic memory in individuals with high-functioning ASD may indeed serve them as a compensatory mechanism for episodic AM. Further research is needed to understand the ways in which semantic knowledge becomes integrated into their personal memories and identities.

\section{COGNITIVE CORRELATES OF EPISODIC AM}

Different cognitive functions have been hypothesized to affect AM patterns in ASD. Impairments in executive functions in ASD, viz., the ability to bind and integrate information (Bowler et al., 2014), correlate significantly with
AM performance (Maister et al., 2013; Goddard et al., 2014). Further, AM ability is related to Theory of Mind (Adler et al., 2010; Crane et al., 2013) and emotional understanding (Losh and Capps, 2003); presumably because the ability to understand others' thoughts and feelings may help one understand that one's own experiences change over time. Emergent findings point to a strong relation between difficulties in AM and future thought in ASD, which may be subserved by a common difficulty in binding and constructing mental events (Lind et al., 2014).

Other basic functions of memory, such as visual, verbal and working memory are not associated with AM (Crane et al., 2013; Goddard et al., 2014). And while reduced AM is considered a symptom of depression, depressed mood is not associated with AM in ASD (Crane et al., 2013). Further, though language ability relates to performance on AM tasks (King et al., 2013), difficulties in AM appear to extend beyond language difficulties (Losh and Capps, 2003; Lind et al., 2014).

The relation between AM and selfunderstanding is more complex. In line with known atypicalities in self-knowledge and self-awareness in ASD (Lind, 2010; Tanweer et al., 2010), adults with ASD have been found to extract less meaning from their personal memories (Crane et al., 2010), and were less likely to organize their self-related memories around the self (Crane et al., 2009). These findings suggest a failure to use the self as an effective memory organizational system in ASD. Further research should clarify whether reduced self-concept leads to reduced AM or results from it (Lind et al., 2014).

\section{EPISODIC AM FOR RECENT AND REMOTE EVENTS}

Examining episodic AM for different periods in participants' lifetimes, studies have found that AM in ASD follows typical patterns of memory deterioration-with more specific memories for recent than remote events - in both adults (Crane and Goddard, 2008; Tanweer et al., 2010) and children (Bruck et al., 2007; Goddard et al., 2014; but see Bon et al., 2012). Interestingly, Crane and Goddard (2008) found that adults with ASD lack the typical increase of memories around adolescence 
years (the "reminiscence bump") that is associated with identity-building memories. Further cross-sectional and longitudinal research is needed to replicate and extend these findings.

\section{CONCLUSIONS}

According to Tulving (2002), episodic memory is a "late-developing, and earlydeteriorating past-oriented memory system, more vulnerable than other memory systems to neuronal dysfunction" (p. 5). Thus, it is perhaps not surprising that in autism, episodic AM is reduced in specificity and structural integration. Nevertheless, an examination of the autobiographical narratives of individuals reveals certain unique characteristics that are shaped by their specific difficulties with social-emotional processing and integration, possibly pointing to a differently configured memory system that may require its own descriptive terminology (Mottron et al., 2008). The narratives of individuals with ASD tend to focus on perceptual or semantic details, favoring pre-structured narratives over lived, emotional experiences. And AM impairments are related to underlying difficulties with integration and emotional understanding, but are not consistently related to other psychological functions, such as depressed mood. As our understanding of AM in ASD deepens, it will be necessary to further delineate the ways in which the memory profile of ASD overlaps and differs from that of other neurological conditions (such as depression, amnesia and other developmental disorders), in order to identify unique and common processes of memory in ASD.

The rich findings regarding $\mathrm{AM}$ in ASD call for several additional avenues of research. First, while reduced episodic AM integration suggests atypical engagement of the hippocampus and mPFC during memory processes (Ben Shalom, 2009), and several studies have shown reduced $\mathrm{mPFC}$ engagement during selfrelated processes in ASD (see Uddin, 2011 for a review), no neurobiological study to date has directly examined AM in ASD. Second, it is necessary to further examine the complex relation between AM, self-understanding and identity, both through careful experimental research that disentangles these components, and through further naturalistic studies that seek to determine how they are integrated. Finally, more longitudinal research, especially around the "reminiscence bump" in adolescence, is needed in order to track the developmental trajectory of semantic and episodic AM in ASD (Bon et al., 2012). Together, these studies will enable us to develop subtle interventions to strengthen the memory integration of individuals with autism, while maintaining their unique characteristics and supporting their self-identity.

\section{ACKNOWLEDGMENTS}

I would like to thank Judith Piggot, Lawrie McKay, Tiffany Wong, Tal Galili, Kristen Gillespie and Michal Govrin for their ongoing collegiality and support. Support during the time of writing was provided by a fellowship from the Israel Ministry of Science and Ministry of Immigrant Absorption.

\section{REFERENCES}

Adler, N., Nadler, B., Eviatar, Z., and ShamayTsoory, S. G. (2010). The relationship between theory of mind and autobiographical memory in high-functioning autism and Asperger syndrome. Psychiatry Res. 178, 214-216. doi: 10.1016/j.psychres.2009.11.015

Bang, J., Burns, J., and Nadig, A. (2013). Brief report: conveying subjective experience in conversation: production of mental state terms and personal narratives in individuals with high functioning autism. J. Autism Dev. Disord. 43, 1732-1740. doi: 10.1007/s10803-012-1716-4

Ben Shalom, D. (2009). The medial prefrontal cortex and integration in autism. Neuroscientist 15, 589-598. doi: 10.1177/1073858409336371

Ben Shalom, D., Faran, Y., and Boucher, J. (2010). A quantitative measure of JS's memory. Autism 14, 619-628. doi: 10.1177/1362361310373704.

Bon, L., Baleyte, J. M., Piolino, P., Desgranges, B., Eustache, F., and Guillery-Girard, B. (2012). Growing up with asperger's syndrome: developmental trajectory of autobiographical memory. Front. Psychol. 3:605. doi: 10.3389/fpsyg.2012. 00605

Boucher, J. (2007). Memory and generativity in very high functioning autism. Autism 11, 255-264. doi: $10.1177 / 1362361307076863$

Boucher, J., and Bowler, D. (eds.). (2008). Memory in Autism: Theory and Evidence. Cambridge: Cambridge University Press. doi: 10.1017/CBO97 80511490101

Boucher, J., Mayes, A., and Bigham, S. (2012). Memory in autistic spectrum disorder. Psychol. Bull. 138, 458-496. doi: 10.1037/a0026869

Bowler, D. M., Gaigg, S. B., and Gardiner, J. M. (2014). Binding of multiple features in memory by high-functioning adults with autism spectrum disorder. J. Autism Dev. Disord. 44, 2355-2362. doi: 10.1007/s10803-014-2105-y
Brezis, R. (2012). “Autism as a case for neuroanthropology: delineating the role of theory of mind in religious development," in The Encultured Brain, eds D. H. Lende and G. Downey (Boston: MIT Press), 291-314.

Brezis, R. S., Galili, T., Wong, T., and Piggot, J. I. (2014). Impaired social processing in autism and its reflections in memory: a deeper view of encoding and retrieval processes. J. Autism Dev. Disord. 44, 1183-1192. doi: 10.1007/s10803-013-1980-y

Brezis, R. S., Wong, T., Pham, D., Galili, T. D., and Piggot, J. (2012). "Autobiographical and social memory narratives in autism: delineating the role of the hippocampus and amygdala," in International Meeting for Autism Research (Toronto, ON).

Brown, B., Morris, G., Nida, R., and Baker-Ward, L. (2012). Brief report: making experience personal: internal states language in the memory narratives of children with and without asperger's disorder. J. Autism Dev. Disord. 42, 441-446. doi: 10.1007/s10803-011-1246-5

Bruck, M., London, K., Landa, R., and Goodman, J. (2007). Autobiographical memory and suggestibility in children with autism spectrum disorder. Dev. Psychopathol. 19, 73-95. doi: 10.1017/S0954579407070058

Chaput, V., Amsellem, F., Urdapilleta, I., Chaste, P., Leboyer, M., Delorme, R., et al. (2013). Episodic memory and self-awareness in Asperger Syndrome: analysis of memory narratives. Res. Autism Spectr. Disord. 7, 1062-1067. doi: 10.1016/ j.rasd.2013.05.005

Crane, L., and Goddard, L. (2008). Episodic and semantic autobiographical memory in adults with autism spectrum disorders. J. Autism Dev. Disord. 38, 498-506. doi: 10.1007/s10803-007-0420-2

Crane, L., Goddard, L., and Pring, L. (2009). Specific and general autobiographical knowledge in adults with autism spectrum disorders: the role of personal goals. Memory 17, 557-576. doi: 10.1080/09658210902960211

Crane, L., Goddard, L., and Pring, L. (2010). Brief report: self-defining and everyday autobiographical memories in adults with autism spectrum disorders. J. Autism Dev. Disord. 40, 383-391. doi: 10.1007/s10803-009-0875-4

Crane, L., Goddard, L., and Pring, L. (2013). Autobiographical memory in adults with autism spectrum disorder: the role of depressed mood, rumination, working memory and theory of mind. Autism 17, 205-219. doi: $10.1177 / 1362361311418690$

Crane, L., Pring, L., Jukes, K., and Goddard, L. (2012). Patterns of autobiographical memory in adults with Autism spectrum disorder. J. Autism Dev. Disord. 42, 2100-2112. doi: 10.1007/s10803-0121459-2

Frith, U., and Happé, F. (1999). Theory of mind and self-consciousness: what is it like to be autistic? Mind Lang. 14, 82-89. doi: 10.1111/14680017.00100

Gardiner, J. M. (2008). "Concepts and theories of memory," in Memory in Autism: Theory and Evidence, eds J. Boucher and D. Bowler (Cambridge: Cambridge University Press), 3-20. doi: 10.1017/CBO9780511490101.003

Gilboa, A. (2004). Autobiographical and episodic memory-one and the same: Evidence from 
prefrontal activation in neuroimaging studies. Neuropsychologia 42, 1336-1349. doi: 10.1016/j. neuropsychologia.2004.02.014

Goddard, L., Dritschel, B., Robinson, S., and Howlin, P. (2014). Development of autobiographical memory in children with autism spectrum disorders: deficits, gains, and predictors of performance. Dev. Psychopathol. 26, 215-228. doi: 10.1017/S0954579413000904

Goddard, L., Howlin, P., Dritschel, B., and Patel, T. (2007). Autobiographical memory and social problem-solving in Asperger syndrome. J. Autism Dev. Disord. 37, 291-300. doi: 10.1007/s10803006-0168-0

Goldman, S. (2008). Brief report: narratives of personal events in children with autism and developmental language disorders: unshared memories. J. Autism Dev. Disord. 38, 1982-1988. doi: 10.1007/s10803-008-0588-0

Hurlburt, R. T., Happé, F., and Frith, U. (1994). Sampling the form of inner experience in three adults with Asperger syndrome. Psychol. Med. 24, 385-395. doi: 10.1017/S0033291700 027367

King, D., Dockrell, J. E., and Stuart, M. (2013). Event narratives in 11-14 year olds with autistic spectrum disorder. Int. J. Lang. Commun. Disord. 48, 522-533. doi: 10.1111/1460-6984.12025

Klein, S. B., Chan, R. L., and Loftus, J. (1999). Independence of episodic and semantic selfknowledge: the case from autism. Social Cogn. 17, 413-436. doi: 10.1521/soco.1999.17.4.413

Lind, S. E. (2010). Memory and the self in autism. Autism 14, 430-456. doi: 10.1177/1362 361309358700

Lind, S. E., Williams, D. M., Bowler, D. M., and Peel, A. (2014). Episodic memory and episodic future thinking impairments in high-functioning autism spectrum disorder: an underlying difficulty with scene construction or self-projection? Neuropsychology 28, 55-67. doi: 10.1037/neu00 00005

Losh, M., and Capps, L. (2003). Narrative ability in high-functioning children with autism or Asperger's syndrome. J. Autism Dev. Disord. 33, 239-251. doi: 10.1023/A:1024446215446
Losh, M., and Capps, L. (2006). Understanding of emotional experience in autism: insights from the personal accounts of high-functioning children with autism. Dev. Psychol. 42, 809-818. doi: 10.1037/0012-1649.42.5.809

Maister, L., Simons, J. S., and Plaisted-Grant, K. (2013). Executive functions are employed to process episodic and relational memories in children with autism spectrum disorders. Neuropsychology 27, 615-627. doi: 10.1037/a0034492

McCabe, A., Hillier, A., and Shapiro, C. (2013). Brief report: structure of personal narratives of adults with autism spectrum disorder. J. Autism Dev. Disord. 43, 733-738. doi: 10.1007/s10803012-1585-x

Mottron, L., Dawson, M., and Soulières, I. (2008). "A different memory: are distinctions drawn from the study of non-autistic memory appropriate to describe memory in autism?" in Memory in Autism: Theory and Evidence, eds J. Boucher and D. Bowler (Cambridge: Cambridge University Press), 311-329. doi: 10.1017/CBO9780511490 101.018

Nickrenz, E. (2007). "Compelling structures: the "Special Interest" in the life-tellings of young adults with Asperger's syndrome," in Presentation at Society for Psychological Anthropology Biennial Meeting (Manhattan Beach, CA).

Piolino, P., Desgranges, B., and Eustache, F. (2009). Episodic autobiographical memories over the course of time: cognitive, neuropsychological and neuroimaging findings. Neuropsychologia 47, 2314-2329. doi: 10.1016/j.neuropsychologia.2009. 01.020

Preston, A. R., and Eichenbaum, H. (2013). Interplay of hippocampus and prefrontal cortex in memory. Curr. Biol. 23, R764-R773. doi: 10.1016/j.cub.2013.05.041

Sirota, K. G. (2010). Narratives of distinction: personal life narrative as a technology of the self in the everyday lives and relational worlds of children with autism. Ethos 38, 93-115. doi: 10.1111/j.15481352.2009.01083.x

Skirrow, P., Jackson, P., Perry, E., and Hare, D. J. (2014). I collect therefore I am-autonoetic consciousness and hoarding in asperger syndrome.
Clin. Psychol. Psychother. doi: 10.1002/cpp.1889. [Epub ahead of print].

Solomon, O. (2004). Narrative introductions: discourse competence of children with autistic spectrum disorders. Discourse Stud. 6, 253-276. doi: 10.1177/1461445604041770

Souchay, C., Guillery-Girard, B., Pauly-Takacs, K., Wojcik, D. Z., and Eustache, F. (2013). Subjective experience of episodic memory and metacognition: a neurodevelopmental approach. Front. Behav. Neurosci. 7:212. doi: 10.3389/fnbeh.2013. 00212

Tanweer, T., Rathbone, C. J., and Souchay, C. (2010). Autobiographical memory, autonoetic consciousness, and identity in Asperger syndrome. Neuropsychologia 48, 900-908. doi: 10. 1016/j.neuropsychologia.2009.11.007

Tulving, E. (2002). EPISODIC MEMORY: from mind to brain. Annu. Rev. Psychol. 53, 1-25. doi: 10.1146/annurev.psych.53.100901.135114

Uddin, L. Q. (2011). The self in autism: an emerging view from neuroimaging. Neurocase 17, 201-208. doi: $10.1080 / 13554794.2010 .509320$

Conflict of Interest Statement: The author declares that the research was conducted in the absence of any commercial or financial relationships that could be construed as a potential conflict of interest.

Received: 15 November 2014; accepted: 30 January 2015; published online: 13 February 2015.

Citation: Brezis RS (2015) Memory integration in the autobiographical narratives of individuals with autism. Front. Hum. Neurosci. 9:76. doi: 10.3389/ fnhum.2015.00076

This article was submitted to the journal Frontiers in Human Neuroscience.

Copyright (c) 2015 Brezis. This is an open-access article distributed under the terms of the Creative Commons Attribution License (CC BY). The use, distribution or reproduction in other forums is permitted, provided the original author(s) or licensor are credited and that the original publication in this journal is cited, in accordance with accepted academic practice. No use, distribution or reproduction is permitted which does not comply with these terms. 